phys. stat. sol. (b) 180, 303 (1993)

Subject classification: $61.70 ; 62.20$

Division of Engineering, Brown University, Providence ${ }^{1}$ )

\title{
On the Nucleation of Dislocations at a Crystal Surface
}

\author{
By \\ G. E. Beltz ${ }^{2}$ ) and L. B. Freund
}

\begin{abstract}
An exact expression for the elastic energy associated with a semicircular shear dislocation loop emanating from a free surface is derived (within continuum dislocation theory) and compared with an earlier approximation. The energy required to activate a semicircular dislocation loop into its unstable "saddle-point" configuration is then re-calculated, based on the modified expression for the self-energy. It is found that the shear stress necessary to emit the loop, as a function of temperature, is almost $50 \%$ less than earlier estimates. The principal drawback to this type of calculation is also discussed, namely, that the critical radius of an incipient dislocation loop is on the order of one atomic spacing, which is too small for a continuum theory to be valid.
\end{abstract}

Ein exakter Ausdruck für die elastische Energie eines halbkreisförmigen Scherversetzungsringes, der von einer freien Oberfläche ausgeht, wird im Rahmen der Kontinuumsversetzungstheorie berechnet und mit früheren Näherungen verglichen. Die Aktivierungsenergie, um einen halbkreisförmigen Versetzungsring in die instabile Sattelpunktskonfiguration zu bringen, wird aufgrund des modifizierten Ausdrucks für die Selbstenergie neu berechnet. Man findet, daß die zur Emittierung eines Ringes notwendige Schubspannung als Funktion der Temperatur etwa $50 \%$ niedriger ist als früher angenommen. Die prinzipielle Schwachstelle dieser Berechnungsweise wird auch diskutiert, nämlich, daß der kritische Radius eines anfänglichen Versetzungsringes in der Größenordnung eines Atomabstandes liegt, was zu klein ist für eine hier noch gültige Kontinuumstheorie.

\section{Introduction}

The behavior of dislocations in materials with electronic applications has been a topic of long-standing interest, because of the effect of dislocations on electrical properties. One example is the appearance of misfit dislocations in layers which have been epitaxially grown onto a substrate with a slightly different lattice parameter. Invariably, this process involves materials which have been chosen primarily for their electronic properties, such as the band-gap width, the resistivity, and the type of charge carrier, and not for reasons related to their lattice parameters. The resulting strain that occurs is commonly relieved by the formation of misfit dislocations. If these dislocations are prevented from forming, the stress in the film is not necessarily detrimental - the stress affects the electronic properties in a predictable manner and is often controlled by exploiting differences in lattice parameters, and subsequently changes in temperature, via a difference in thermal expansion coefficients.

The appearance of misfit dislocations during epitaxial growth has been observed experimentally to coincide with the attainment of a critical thickness, which in turn depends

1) Providence, RI 02912, USA.

2) Affiliation until January, 1994: Max-Planck-Institut für Metallforschung, Institut für Werkstoffwissenschaft, Seestraße 92, D-70174 Stuttgart, Federal Republic of Germany; affiliation as of January, 1994: Department of Mechanical and Environmental Engineering, University of California, Santa Barbara, CA 93106, USA. 
on the misfit strain, elastic constants, and the orientation of the various slip systems involved (e.g., Matthews [1], Hull et al. [2, 3], and Houghton et al. [4]). Theoretical studies of this problem have established the validity of the critical thickness concept, starting with Frank and van der Merwe [5,6] and leading up to recent work by Freund [7, 8]. Most analyses to date, however, have concentrated on analyzing the stability of a single pre-existing threading dislocation, or a threading dislocation in the presence of an array of other dislocations. Another issue that must be addressed more rigorously is the actual nucleation of dislocations. If dislocations could somehow be prevented from forming to begin with during the epitaxial growth process, then results pertaining to fully formed dislocations would not be as relevant.

A possible location at which dislocations are actually generated during film growth is the film surface. The primary purpose of this paper is to re-examine the process of dislocation nucleation at a crystal surface, in light of recent developments with the theory of dislocation formation. First, an expression for the shear stress due to a general shear dislocation loop perpendicular to a free surface is derived, and immediately utilized to derive an accurate expression for the elastic self-energy of a semicircular loop. Next, conditions for nucleation of the semicircular loop are worked out in the continuum framework described by Hirth [9] and subsequently by Fitzgerald et al. [10], but with the modified expression for the self-energy of the loop. Finally, the potential utility of the expression for shear stress derived here in more atomistic-type calculations is pointed out.

\section{The Energy of a Semicircular Dislocation Loop at a Free Surface}

We present here a calculation of the self-energy of a dislocation loop emerging perpendicularly from a free surface in an isotropic, elastic half-space. To understand the nature of the principal result of this section, recall that the elastic self-energy for a full circular dislocation loop of radius $r$ in an infinite elastic solid is given by [11]

$$
U^{\mathrm{full}}=\frac{\mu b^{2} r}{4} \frac{(2-v)}{(1-v)} \ln \left(\frac{8 r}{e^{2} r_{0}}\right),
$$

where $\mu$ is the shear modulus, $b$ the Burgers vector, $v$ Poisson's ratio, and $r_{0}$ the core cut-off radius. In analogous fashion to what Gao and Rice [12] have done for a general dislocation loop ahead of a crack, we will show here that the energy of the semicircular loop of radius $r$ emerging from the free surface has the form

$$
U^{\text {half }}=\frac{\mu b^{2} r}{8} \frac{(2-v)}{(1-v)} \ln \left(\frac{8 m r}{e^{2} r_{0}}\right),
$$

where $m$ is a geometry-dependent correction factor. Early analyses of dislocation loop nucleation at cracks [13], as well as most analyses of nucleation at a free surface to date $[9,10,14]$, begin with the assumption that $m=1$, i.e., by estimating $U^{\text {half }}$ as half the energy of a full circular loop.

Simple physical arguments may be invoked to state that $0<m<1$. The former inequality is expected because the energy becomes unbounded as $m \rightarrow 0$. The latter inequality follows from considering the formation of a full circular dislocation loop in an infinite solid by bonding together two half-spaces, each containing a semicircular loop with the same radius (see Fig. 1). The resultant energy must be the sum of the two energies of the half-space 

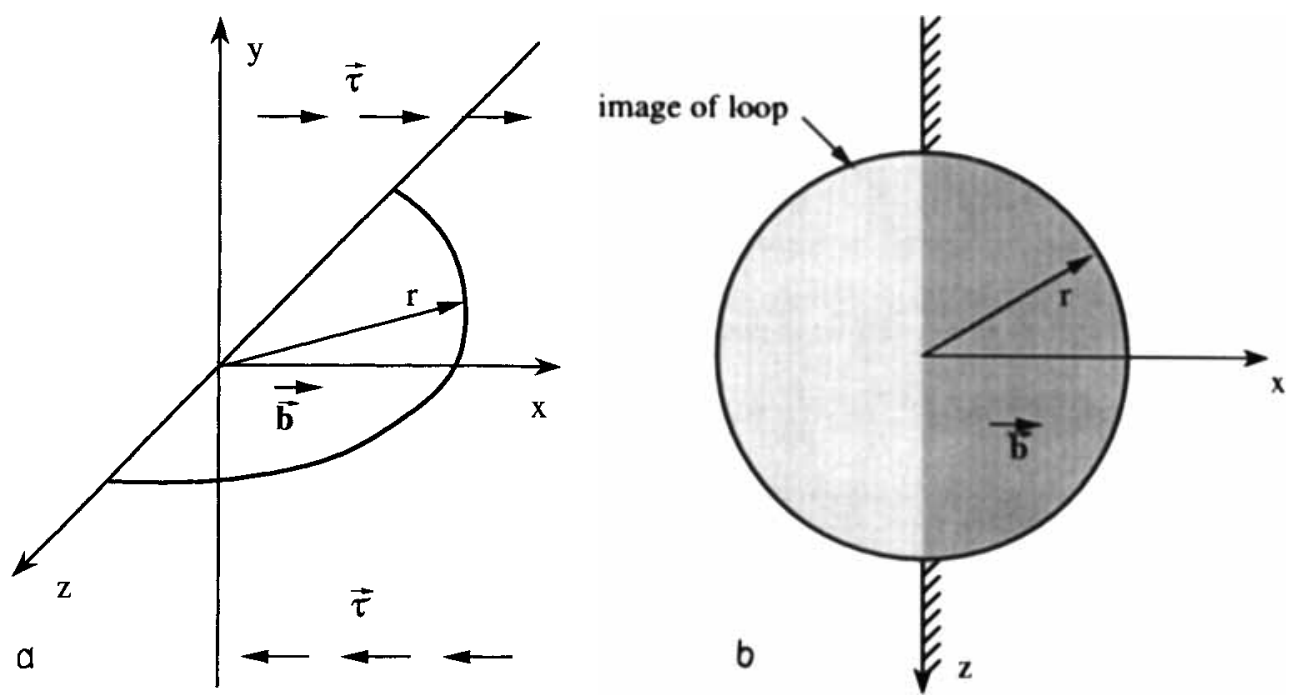

Fig. 1. Geometry of an emergent semicircular shear dislocation loop at a free surface. The slip plane, as well as the Burgers vector, are perpendicular to the surface

configurations, as well as the positive work that must be done on the free surfaces to make the two spaces match. I.e., the inequality $2 U^{\text {half }}<U^{\text {full }}$ must hold, which is consistent with $m<1$.

The calculation of $m$ follows the procedure outlined by Gao and Rice [12] and Anderson and Rice [15] in that (1) and (2) may be combined and re-arranged to give the following expression for $m$ :

$$
\ln (m)=\frac{8}{\mu b^{2} r} \frac{(1-v)}{(2-v)}\left(U^{\text {half }}-\frac{1}{2} U^{\mathrm{full}}\right) .
$$

The difference in elastic energy between the dislocation emanating from the free surface and one half that of the corresponding full loop in an infinite body is calculated by integrating the corresponding difference in work done in forming the dislocations, $-(b / 2)\left[\tau^{\text {half }}(x, 0, z)\right.$ $\left.-\tau^{\mathrm{full}}(x, 0, z)\right]$, over the entire area of the half-loop. The integral of the stress difference converges, even though the limits of integration extend entirely up to the dislocation line itself. Hence, an expression may be written for $m$ that is independent of any core cutoff procedure,

$$
\ln (m)=-\frac{4}{\mu b r} \frac{(1-v)}{(2-v)} \int_{A}\left[\tau^{\text {half }}(x, 0, z)-\tau^{\mathrm{full}}(x, 0, z)\right] \mathrm{d} A,
$$

where $\tau^{\text {half }}$ and $\tau^{\text {full }}$ are, respectively, exact expressions (within continuum elastic dislocation theory) for the shear stress of the half-loop at the free surface and the full loop in an infinite solid. As will be evident later, a common factor of $r$ appears upon non-dimensionalization of the integral in (4). Hence, $m$ should depend only on $v$.

For now, assume that a general Burgers displacement $\delta(x, z)$ (defined as $u_{x}^{+}(x, z)$ $-u_{x}^{-}(x, z)$, where $u_{x}^{+}$and $u_{x}^{-}$denote the displacement immediately above and below the 
slip plane) exists on a slip plane, for purposes of discussing expressions for the shear stresses needed for evaluating (4). Later, $\delta(x, z)$ will be replaced by $b$ in a circular dislocated region. The shear stress $\tau^{\text {full }}(x, 0, z)$ associated with a dislocation loop in an infinite solid is $[12,16,17]$

$$
\tau^{\mathrm{full}}(x, 0, z)=\frac{\mu}{4 \pi(\dot{1}-v)} \int_{-\infty}^{\infty} \int_{-\infty}^{\infty} K^{\infty}(x, z ; \tilde{x}, \tilde{z}) \delta(\tilde{x}, \tilde{z}) \mathrm{d} \tilde{x} \mathrm{~d} \tilde{z},
$$

where the operator $K^{\infty}(x, z ; \tilde{x}, \tilde{z})$ is given by

$$
K^{\infty}(x, z ; \tilde{x}, \tilde{z}) \equiv \frac{\tilde{x}-x}{D^{3}} \frac{\partial}{\partial \tilde{x}}+(1-v) \frac{\tilde{z}-z}{D^{3}} \frac{\partial}{\partial \tilde{z}}
$$

and $D \equiv \sqrt{(x-\tilde{x})^{2}+(z-\tilde{z})^{2}}$. The domain of integration may be restricted to the dislocated region, i.e., where $\delta(x, z)$ is nonzero. However, care must be exercised at the boundaries if, for example, the gradient of $\delta(x, z)$ is Dirac-singular.

The derivation of an expression for the shear stresses around a dislocated region in a half-space, $\tau^{\text {half }}(x, 0, z)$, is somewhat more involved and follows the framework laid out by Bacon and Groves [18] and Eshelby [19]. In that formalism, the infinitesimal displacement field due to an infinitesimal dislocation loop located at $(\tilde{x}, 0, \tilde{z})$ is written as

$$
\mathrm{d} u_{i}(x, y, z ; \tilde{x}, \tilde{z})=\mathrm{d} u_{i}^{\infty}(x, y, z ; \tilde{x}, \tilde{z})+\mathrm{d} u_{i}^{\mathrm{I}}(x, y, z ; \tilde{x}, \tilde{z})+\mathrm{d} u_{i}^{\mathrm{S}}(x, y, z ; \tilde{x}, \tilde{z}),
$$

where $\mathrm{d} u_{i}^{\infty}(x, y, z ; \tilde{x}, \tilde{z})$ are the displacements due to the loop at $(\tilde{x}, 0, \tilde{z})$ in an infinite body, $\mathrm{d} u_{i}^{\mathrm{l}}(x, y, z ; \tilde{x}, \tilde{z})$ are the displacements due to a loop of opposite sign at $(-\tilde{x}, 0 \tilde{z})$ in an infinite medium (the so-called image term), and $\mathrm{d} u_{i}^{\mathrm{S}}(x, y, z ; \tilde{x}, \tilde{z})$ are the displacements required to make the surface traction-free. The subscript $i$ refers the displacement component to the $x-, y$-, or $z$-direction. The shear stress associated with the finite dislocation loop in a half-space may then be obtained by integration of the displacements in (6) over the loop area, differentiation to determine the strains, and finally application of Hooke's law. Fortunately, the result is already known for the contributions from $\mathrm{d} u_{i}^{\infty}(x, y, z)$ and $\mathrm{d} u_{i}^{\mathrm{I}}(x, y, z)$, by making appropriate use of (5). If the shear stress is broken into three corresponding contributions,

$$
\tau^{\text {half }}(x, 0, z)=\tau^{\infty}(x, 0, z)+\tau^{\mathrm{I}}(x, 0, z)+\tau^{\mathrm{S}}(x, 0, z),
$$

then $\tau^{\infty}(x, 0, z)$ and $\tau^{\mathrm{I}}(x, 0, z)$ may be written as

$$
\begin{aligned}
\tau^{\infty}(x, 0, z) & =\frac{\mu}{4 \pi(1-v)} \int_{-\infty}^{\infty} \int_{0^{-}}^{\infty} K^{\infty}(x, z ; \tilde{x}, \tilde{z}) \delta(\tilde{x}, \tilde{z}) \mathrm{d} \tilde{x} \mathrm{~d} \tilde{z}
\end{aligned}
$$

where the operator $K^{1}(x, z ; \tilde{x}, \tilde{z})$ is given by

$$
K^{\mathrm{I}}(x, z ; \tilde{x}, \tilde{z}) \equiv \frac{\tilde{x}+x}{R^{3}} \frac{\partial}{\partial \tilde{x}}+(1-v) \frac{\tilde{z}-z}{R^{3}} \frac{\partial}{\partial \tilde{z}}
$$


and $R \equiv \sqrt{(x+\tilde{x})^{2}+(z-\tilde{z})^{2}}$. Again, care must be taken with the limits of integration. In the half-space, the dislocation loop terminates as a line along the free surface, i.e., the gradient of $\delta(x, z)$ is generally Dirac-singular along $x=0$. In anticipation of a numerical problem due to a $1 / x$ singularity at the free surface, the contribution to the integrals in $\tilde{x}$ between $0^{-}$and $0^{+}$is evaluated, giving

$$
\begin{aligned}
\tau^{\infty}(x, 0, z)= & -\frac{\mu x}{4 \pi(1-v)} \int_{-\infty}^{\infty} \frac{\delta\left(0^{+}, \tilde{z}\right) \mathrm{d} \tilde{z}}{\left[x^{2}+(z-\tilde{z})^{2}\right]^{3 / 2}} \\
& +\frac{\mu}{4 \pi(1-v)} \int_{-\infty}^{\infty} \int_{0^{+}}^{\infty} K^{\infty}(x, z ; \tilde{x}, \tilde{z}) \delta(\tilde{x}, \tilde{z}) \mathrm{d} \tilde{x} \mathrm{~d} \tilde{z}, \\
\tau^{\mathrm{I}}(x, 0, z)= & -\frac{\mu x}{4 \pi(1-v)} \int_{-\infty}^{\infty} \frac{\mu\left(0^{+}, \tilde{z}\right) \mathrm{d} \tilde{z}}{\left[x^{2}+(z-\tilde{z})^{2}\right]^{3 / 2}} \\
& -\frac{\mu}{4 \pi(1-v)} \int_{-\infty}^{\infty} \int_{0^{+}}^{\infty} K^{\mathrm{I}}(x, z ; \tilde{x}, \tilde{z}) \delta(\tilde{x}, \tilde{z}) \mathrm{d} \tilde{x} \mathrm{~d} \tilde{z} .
\end{aligned}
$$

The infinitesimal displacement expressions $\mathrm{d} u_{i}^{\mathrm{s}}(x, y, z)$ for a shear loop perpendicular to a free surface are $[18,19]$

$$
\begin{aligned}
& \mathrm{d} u_{x}^{\mathrm{s}}(x, y, z ; \tilde{x}, \tilde{z})=\frac{b \tilde{x} \mathrm{~d} S}{4 \pi(1-v)}\left\{(4-2 v) \frac{\partial^{2}}{\partial x \partial y}\left(\frac{1}{\check{\tilde{R}}}\right)-\frac{\partial^{3}}{\partial x^{2} \partial y}\left(\frac{x}{\check{R}}\right)\right\}, \\
& \mathrm{d} u_{y}^{\mathrm{s}}(x, y, z ; \tilde{x}, \tilde{z})=\frac{b \tilde{x} \mathrm{~d} S}{4 \pi(1-v)}\left\{2 v \frac{\partial^{2}}{\partial x \partial y}\left(\frac{1}{\check{R}}\right)-\frac{\partial^{3}}{\partial x \partial y^{2}}\left(\frac{x}{\check{R}}\right)\right\},
\end{aligned}
$$

where $\check{R} \equiv \sqrt{(x+\tilde{x})^{2}+(z-\tilde{z})^{2}+y^{2}}$. Equations (10a) and (10b) may be differentiated and combined to obtain the shear strain

$$
\mathrm{d} \varepsilon_{x y}=\frac{1}{2} \mathrm{~d}\left(\frac{\partial u_{y}}{\partial x}+\frac{\partial u_{x}}{\partial y}\right)
$$

and the shear stress is determined via Hooke's law, i.e.,

$$
\mathrm{d} \tau_{x y}=2 \mu \mathrm{d} \varepsilon_{x y} .
$$

The final expression is found by integrating over the loop area and evaluating at $y=0$ to obtain

$$
\tau^{\mathrm{S}}(x, 0, z)=\frac{2 \mu x}{4 \pi(1-v)} \int_{-\infty}^{\infty} \int_{0}^{\infty}\left[\frac{15 \tilde{x}(\tilde{x}+x)^{2}}{R^{7}}-\frac{3 \tilde{x}}{R^{5}}\right] \delta(\tilde{x}, \tilde{z}) \mathrm{d} \tilde{x} \mathrm{~d} \tilde{z} .
$$


The integrand was verified by means of a symbolic manipulation program. The final expression for $\tau^{\text {half }}(x, 0, z)$ is determined by combining (9) and (13) to give

$$
\begin{aligned}
\tau^{\text {half }}(x, 0, z)= & -\frac{2 \mu x}{4 \pi(1-v)} \int_{-\infty}^{\infty} \frac{\delta\left(0^{+}, \tilde{z}\right) \mathrm{d} \tilde{z}}{\left[x^{2}+(z-\tilde{z})^{2}\right]^{3 / 2}} \\
& +\frac{\mu}{4 \pi(1-v)} \int_{-\infty}^{\infty} \int_{0^{+}}^{\infty}\left[K^{\infty}(x, z ; \tilde{x}, \tilde{z})-K^{\mathrm{I}}(x, z ; \tilde{x}, \tilde{z})\right] \delta(\tilde{x}, \tilde{z}) \mathrm{d} \tilde{x} \mathrm{~d} \tilde{z} \\
& +\frac{2 \mu x}{4 \pi(1-v)} \int_{-\infty}^{\infty} \int_{0}^{\infty}\left[\frac{15 \tilde{x}(\tilde{x}+x)^{2}}{R^{7}}-\frac{3 \tilde{x}}{R^{5}}\right] \delta(\tilde{x}, \tilde{z}) \mathrm{d} \tilde{x} \mathrm{~d} \tilde{z} .
\end{aligned}
$$

Following [12] and [15], the Cauchy singularities in the integrands may be combined by subtracting out a uniform displacement that has the effect of making the integrand finite as $\tilde{x}$ approaches 0 ,

$$
\begin{aligned}
\tau^{\text {half }}(x, 0, z)= & -\frac{2 \mu x}{4 \pi(1-v)} \int_{-\infty}^{\infty} \frac{\delta\left(0^{+}, \tilde{z}\right)-\delta\left(0^{+}, z\right)}{\left[x^{2}+(z-\tilde{z})^{2}\right]^{3 / 2}} \mathrm{~d} \tilde{z} \\
& +\frac{\mu}{4 \pi(1-v)} \int_{-\infty}^{\infty} \int_{0^{+}}^{\infty}\left[K^{\infty}(x, z ; \tilde{x}, \tilde{z})-K^{\mathrm{I}}(x, z ; \tilde{x}, \tilde{z})\right] \delta(\tilde{x}, \tilde{z}) \mathrm{d} \tilde{x} \mathrm{~d} \tilde{z} \\
& +\frac{2 \mu x}{4 \pi(1-v)} \int_{-\infty}^{\infty} \int_{0}^{\infty}\left[\frac{15 \tilde{x}(\tilde{x}+x)^{2}}{R^{7}}-\frac{3 \tilde{x}}{R^{5}}\right] \\
& \times\left\{\delta(\tilde{x}, \tilde{z})-\delta\left(0^{+}, z\right)\right\} \mathrm{d} \tilde{x} \mathrm{~d} \tilde{z} .
\end{aligned}
$$

The result gives the shear stress induced for an arbitrary opening $\delta(x, z)$.

The integrand in (4) may now be formulated by combining (5) and (15) to give

$$
\begin{aligned}
\tau^{\text {half }}-\tau^{\text {full }}= & -\frac{2 \mu x}{4 \pi(1-v)} \int_{-\infty}^{\infty} \frac{\delta\left(0^{+}, \tilde{z}\right)-\delta\left(0^{+}, z\right)}{\left[x^{2}+(z-\tilde{z})^{2}\right]^{3 / 2}} \mathrm{~d} \tilde{z} \\
& -\frac{2 \mu}{4 \pi(1-v)} \int_{-\infty}^{\infty} \int_{0^{+}}^{\infty} K^{1}(x, z ; \tilde{x}, \tilde{z}) \delta(\tilde{x}, \tilde{z}) \mathrm{d} \tilde{x} \mathrm{~d} \tilde{z} \\
& +\frac{2 \mu x}{4 \pi(1-v)} \int_{-\infty}^{\infty} \int_{0}^{\infty}\left[\frac{15 \tilde{x}(\tilde{x}+x)^{2}}{R^{7}}-\frac{3 \tilde{x}}{R^{5}}\right] \\
& \times\left\{\delta(\tilde{x}, \tilde{z})-\delta\left(0^{+}, z\right)\right\} \mathrm{d} \tilde{x} \mathrm{~d} \tilde{z} .
\end{aligned}
$$

The four-fold integral in (4) was carried out numerically via a 21-point Gauss-Kronrod rule with a relative error in the result of $10^{-4}$. The integrand was broken up in such a way 


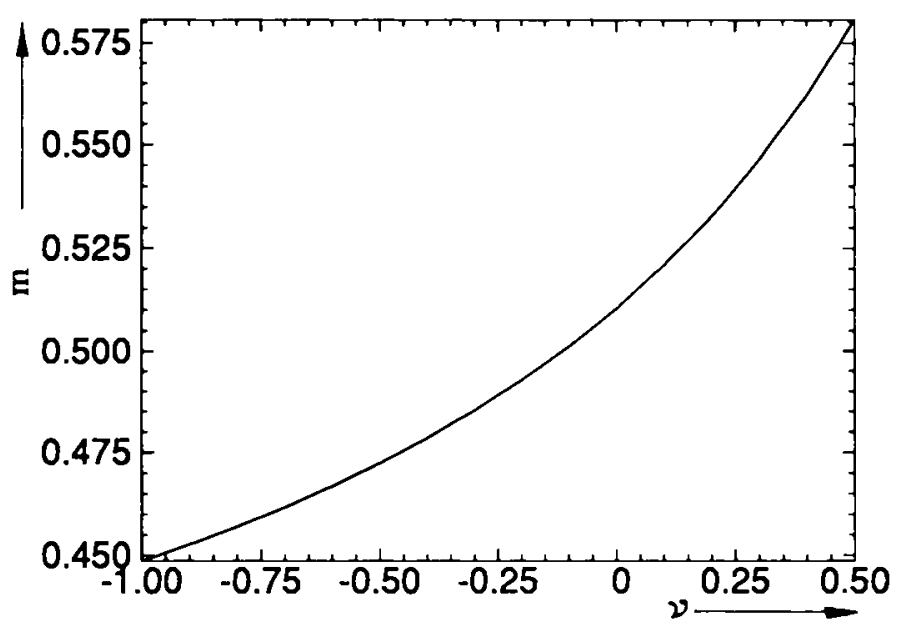

Fig. 2. Plot of the correction factor $m$ as a function of Poisson's ratio $v$

as to give a result that explicitly depends on $v$,

$$
m=\exp \left[\frac{0.7734-1.059(2-v)}{2-v}\right] .
$$

When $v=0.3, m=0.546$ and when $v=0.218$, as appropriate for silicon, $m=0.535$. Fig. 2 shows $m$ as a function of $v$. In principle, analogous procedures to that presented here for the shear loop perpendicular to the free surface may be used to calculate $m$ for general loop shapes, with arbitrary slip plane and Burgers vector orientations.

\section{Nucleation of a Semicircular Loop}

The calculation presented in this section essentially follows that of Hirth [9], in which the energy associated with an incipient semicircular dislocation loop is determined, and then rendered stationary in order to find the critical radius of the loop, beyond which the loop will unstably enlarge. It is assumed that the dislocated plane, as well as the direction of slip, are perpendicular to the crystal surface (as in Fig. 1). The loading is assumed to be a uniform, remotely applied shear stress $\tau$. It is also assumed that the dislocation is not dissociated, i.e., that the stacking fault energy does not contribute in any fashion. The total energy of the loop then consists of three terms,

$$
E=U^{\text {half }}+U^{\text {lcdge }}-W^{\text {stress }}
$$

where $U^{\text {half }}$ is as discussed in the previous section, $U^{\text {ledge }}$ is the energy of the ledge that is created (or removed) at the surface due to the intersecting dislocated plane, and $W^{\text {stress }}$ is the work done by the applied load,

$$
W^{\text {stress }}=\frac{1}{2} \pi r^{2} \tau b
$$

which is the product of the Peach-Koehler force $\tau b$ and its work-conjugate displacement, the area that the dislocation has swept out.

The ledge energy is commonly written as

$$
U^{\text {ledge }}= \pm 2 \gamma b r \text {, }
$$


where the positive value is used for ledge creation, the negative sign is taken for ledge removal, and $\gamma$ is the surface energy. This is a questionable approximation, since it is difficult to define a true thermodynamic surface energy for an area that is one atomic spacing wide. It is perhaps best to interpret $\gamma b$ as an effective ledge energy per unit length. Following [10] and [14], we take $\gamma=\mu b / 8$. The conclusions noted later are relatively insensitive to the choice of this parameter.

At absolute zero, the condition for dislocation emission is given by rendering the total energy stationary,

$$
\begin{aligned}
& \frac{\partial E}{\partial r}=\frac{\mu b^{2}}{8} \frac{(2-v)}{(1-v)} \ln \left(\frac{8 m r}{e r_{0}}\right) \pm 2 \gamma b-\pi r \tau b=0, \\
& \frac{\partial^{2} E}{\partial r^{2}}=\frac{\mu b^{2}}{8 r} \frac{(2-v)}{(1-v)}-\pi \tau b=0,
\end{aligned}
$$

where the latter condition is a stability criterion, i.e., it insures that the energy decreases as the loop expands. Solving for the critical stress for which the loop would spontaneously enlarge gives $\tau_{\text {crit }}^{0}=0.505 \mu$, assuming ledge creation, or $\tau_{\text {crit }}^{0}=0.0873 \mu$, assuming ledge removal. If it is assumed that $m=1$, then the critical stresses increase to $0.944 \mu$ and $0.163 \mu$, respectively. Thus an accurate expression for the self-energy of a loop near the free surface leads to a decrease, by almost a factor of 2 , of the critical stress necessary to homogeneously nucleate the loop. All the calculations presented here use $r_{0}=b / 4$ and $v=0.218$.

The nucleation process, however, realistically occurs at temperatures well above absolute zero. The critical stress for emission may then be determined via an activation energy concept. Assume that the local shear stress is less than $\tau_{\text {crit }}^{0}$ There will then exist two solutions to (21a) - one corresponding to a local minimum of the total energy $\left(r=r_{1}\right)$, and the second corresponding to a local maximum of energy $\left(r=r_{2}\right)$. In general, $r_{2}>r_{1}$. The energy difference between these two states, $\Delta E \equiv E\left(r_{2}\right)-E\left(r_{1}\right)$, defines the activation energy. If energy from thermal vibrations is sufficient to overcome this activation energy,

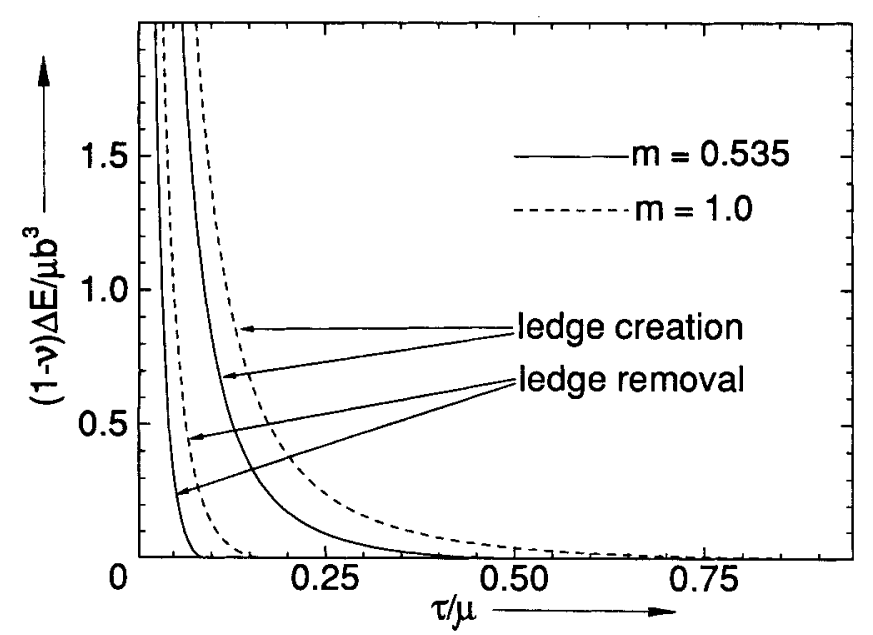

Fig. 3. The activation energy for dislocation loop emission as a function of the applied shear stress 
then the dislocation loop is spontaneously emitted. The activation energy is plotted in Fig. 3 for $m=0.535$ as well as $m=1$. Qualitatively, the behavior is as expected: the energy barrier to dislocation emission decreases as the applied stress approaches its maximum value of $\tau_{\text {crit. }}^{0}$. The decrease in activation energy is relatively sharp for $\tau<\tau_{\text {crit }}^{0}$, but as the limiting stress is approached, the rate of decrease slows, suggesting that thermal vibrations may be sufficient to emit dislocations for a certain stress range below $\tau_{\text {crit }}^{0}$. More importantly, note that using an appropriate value of $m$ leads to significantly reduced activation energies for most loads there is more than a factor of 2 difference. Additionally, note when it is assumed that the emitting dislocation is removing a ledge at the surface, the energy barrier is significantly reduced.

Now that the activation energy required for the emission of a dislocation from the surface is determined, we need some estimate of what energy is available for the process. An estimate by Rice and Beltz [20] is based on a standard Arrhenius relation between the nucleation rate and $\Delta E / k T$ ( $k$ is Boltzmann's factor), and assumes that a "reasonable" nucleation rate is given by one dislocation per second per millimeter of crack front. This elementary calculation leads to an available energy of $43 k T$. A similar argument by Hirth [9] gives $88 k T$ of available energy. Given the uncertainty present in this type of calculation, we follow Fitzgerald et al. [10] and assume that the available energy is $50 k T$. Based on the activation energies in Fig. 3, the stress necessary to emit a dislocation loop as a function of temperature is given in Fig. 4. As expected, a proper choice for $m$ reduces the shear stress by a factor of about 2. Furthermore, the critical stress for a nucleation process associated with removal of a ledge at the surface is about three times less than the stress when the ledge is created.

\section{Validity of the Continuum Approach}

The largest source of uncertainty in the type of calculation presented in the previous section is the fact that a continuum-based description of a dislocation is used over length scales that are much too small. For example, the critical radius for dislocation emission at absolute zero, when a ledge is being removed, is $1.038 b$, according to the theory outlined in the previous section. When a ledge is being created, the critical radius reduces to $0.505 b$ (these numbers assume $m=0.535$ ). In the latter case, the elastic energy predicted by (2) is negative. To realistically calculate the energy associated with a dislocation loop this small, some kind of atomistic theory is needed to model the core.

Furthermore, the expressions used in this paper assume that the dislocation is fully formed. One example of a situation where these considerations have been addressed is the problem of dislocation nucleation at crack tips, which up until recently has also relied on continuum energy expressions for dislocations. Weertman [21] and, more recently, Argon [22] and Schöck [23] have suggested that the Burgers displacement (i.e., the function $\delta(x, z)$ from Section 2), as well as the relative position of the dislocation core, should be regarded as configurational parameters when modeling dislocation nucleation and evaluating the activation energy. A reasonably exact treatment of this phenomenon has been given recently by Rice and coworkers [ 24 to 27] that makes use of the Peierls-Nabarro dislocation model $[28,29]$. In that treatment, the two-dimensional elasticity problem of a traction-free crack with a periodic, nonlinear stress versus displacement relation being satisfied as a boundary condition along a slip plane ahead of the crack tip is solved. Once this interfacial "constitutive" relation is specified (it could be as simple as the Frenkel sinusoidal law), and the elasticity problem solved, there is no need for the core-cutoff parameter (used earlier 


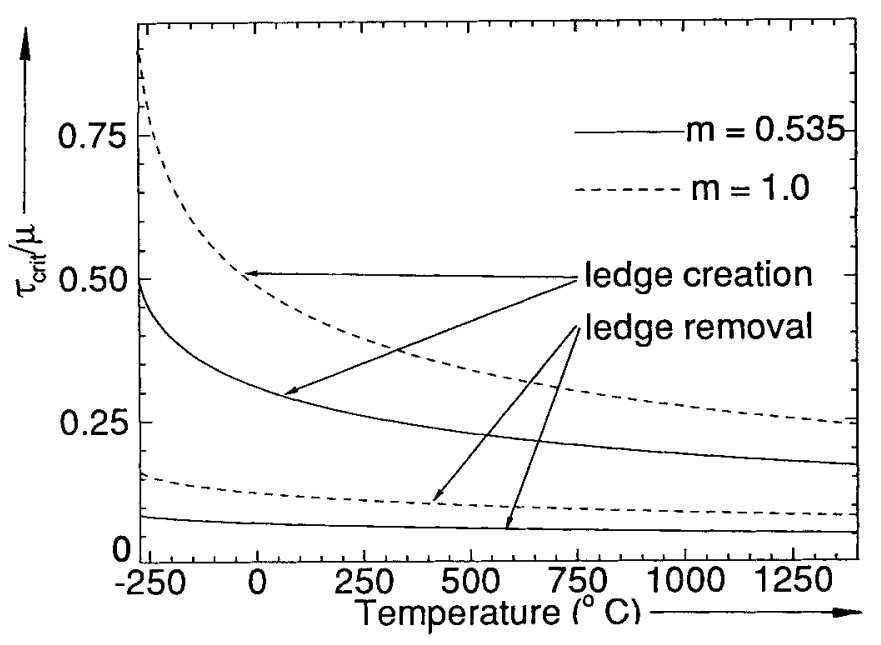

Fig. 4. The critical stress required to emit a dislocation loop as a function of temperature

in this paper). The advantage of this method is that it allows for the existence of an extended dislocation core during nucleation, and eliminates uncertainty involved when using expressions that were derived with the usual core cutoff procedures.

The same types of ideas may be applied to the problem of dislocation nucleation at a surface [30]. Although the problem may be quite straightforwardly worked out for the two-dimensional case (yielding an activation energy per unit length of dislocation parallel to the surface), the three-dimensional case appears to be quite complex. The problem would essentially involve finding a saddle-point dislocation configuration at the surface, i.e., a function $\delta(x, z)$ that renders the total energy stationary. Any numerical solution to this problem within the Peierls-Nabarro framework would necessarily involve a suitable form of (15).

\section{Acknowledgements}

This work has been supported by the Air Force Office of Scientific Research through Grant No. F49620-92-J-0219 and by the Office of Naval Research through Contract No. N00014-90-J-4051. The computations were performed in the Computational Mechanics Research Facility of Brown University.

\section{References}

[1] J. W. Matthews, J. Vacuum Sci. Technol. 12, 126 (1975).

[2] R. Hull, J. C. Bean, J. M. Bonar, and L. Peticolas, Epitaxial Heterostructures, Ed. D. W. Shaw, J. C. Bean, V. G. Keramidas, and P. S. Peercy, Mater. Res. Soc. Symp. Proc., Vol. 198, Mater Res. Soc., Pittsburgh 1990 (p. 459).

[3] R. Hull, J. C. Bean, F. Ross, D. Bahnck, and L. Peticolas, Thin Films: Stresses and Mechanical Properties III, Ed. W. D. Nix, J. C. Bravman, E. Arzt, and L. B. Freund, Mater. Res. Soc. Symp. Proc., Vol. 239, Mater. Res. Soc., Pittsburgh 1992 (p. 379).

[4] D. C. Houghton, D. D. Perovic, G. C. Weatherly, and J.-M. Baribeau, J. appl. Phys. 67, 1850 (1990).

[5] F. C. Frank and J. H. van der Merwe, Proc. Roy. Soc. A 198, 205 (1949).

[6] F. C. Frank and J. H. van der Merwe, Proc. Roy. Soc, A 198, 216 (1949). 
[7] L. B. Freund, J. appl. Mech. 54, 553 (1987).

[8] L. B. Freund, J. Mech. Phys. Solids 38, 657 (1990).

[9] J. P. HiRth, The Relation between the Structure and Mechanical Properties of Metals, Nat. Phys. Lab. Proc., Symp. No. 15, Her Majesty's Stationary Office, London 1963 (p. 218).

[10] E. A. Fitzgerald, G. P. Watson, R. E. Proano, D. G. Ast, P. D. Kirchner, G. D. Petit, and J. M. Woodall, J. appl. Phys. 65, 2220 (1989).

[11] J. P. HiRTH and J. Lothe, Theory of Dislocations, McGraw-Hill, New York 1968 (p. 145).

[12] H. GAO and J. R. RiCE, J. Mech. Phys. Solids 37, 155 (1989).

[13] J. R. Rice and R. Thomson, Phil. Mag. 29, 73 (1974).

[14] J. W. Matthews, A. E. Blakeslee, and S. Mader, Thin Solid Films 33, 253 (1976).

[15] P. M. Anderson and J. R. Rice, J. Mech. Phys. Solids 35, 743 (1987).

[16] R. But, J. Mech. Phys. Solids 25, 29 (1977).

[17] J. WeAvER, Internat. J. Solid and Structures 13, 321 (1977).

[18] D. J. Bacon and P. P. Groves, Fundamental Aspects of Dislocation Theory, Ed. J. A. Simmens, R. DE Wit, and R. Bullough, Vol. 1, Nat. Bur. Standards spec. Publ. 317, Gaithersburg 1969 (p. 35).

[19] J. D. Eshelby, Dislocations in Solids, Ed. F. R. N. Nabarro, Vol. 1, North-Holland Publ. Co., 1979 (p. 167).

[20] J. R. Rice and G. E. Beltz, J. Mech. Phys. Solids, in the press (1994).

[21] J. Weer tMan, Phil. Mag. A43, 1103 (1981).

[22] A. S. ARgON, Acta Metall. 35, 185 (1987).

[23] G. ScHöck, Phil. Mag. A63, 111 (1991).

[24] J. R. Rice, J. Mech. Phys. Solids 40, 239 (1992).

[25] G. E. BeltZ, Ph.D. Thesis, Division of Applied Sciences, Harvard University, Cambridge, Massachusetts (1992).

[26] J. R. Rice, G. E. Beltz, and Y. Sun, Topics in Fracture and Fatigue, Ed. A. S. ARgon, SpringerVerlag, New York 1992 (p. 1).

[27] Y. Sun, G. E. Betrz, and J. R. Rice, Mater. Sci. Engng. A, in press (1993).

[28] R. E. Peierls, Proc. Phys. Soc. 52, 34 (1940).

[29] F. R. N. Nabarro, Proc. Phys. Soc. 59, 256 (1947).

[30] G. E. BeLtZ, work in progress (1993). 